Article

\title{
Silver-catalyzed carboxylative cyclization of alkynic hydrazones with carbon dioxide
}

\author{
Wenzhen Zhang *, Yuqian Sun, Min Zhang, Hui Zhou, Xiaobing Lu \\ State Key Laboratory of Fine Chemicals, Dalian University of Technology, Dalian 110624, Liaoning, China
}

\section{A R T I C L E I N F}

\section{Article history:}

Received 29 January 2019

Accepted 9 March 2019

Published 5 August 2019

\section{Keywords:}

Carbon dioxide

Silver catalysis

Carboxylative cyclization

Alkynic hydrazine

Oxadiazinone

Homogeneous catalysis

\begin{abstract}
A B S T R A C T
The development of new catalytic methodologies to synthesize heterocyclic fine chemicals using carbon dioxide as a synthon has attracted considerable attention. Herein, we report the silver(I)-catalyzed carboxylative cyclization of a variety of alkynic hydrazones with carbon dioxide to produce the corresponding 1,3,4-oxadiazin-2-ones under mild reaction conditions. In this reaction, silver(I) salts play a $\pi$-Lewis acid role for the highly efficient activation of the alkyne moiety in the hydrazone substrates. Single-crystal X-ray analysis and NOE experiments confirm that the newly formed oxadiazinone products exhibit $Z$ configuration. Based on control experiments and NMR studies, a mechanism including the formation of a reactive carbazate intermediate, electrophilic cyclization, and subsequent protonation is proposed. This study offers an efficient and atom-economical method for the synthesis of biologically important 1,3,4-oxadiazin-2-ones.
\end{abstract}

(C) 2019, Dalian Institute of Chemical Physics, Chinese Academy of Sciences. Published by Elsevier B.V. All rights reserved.

\section{Introduction}

The catalytic transformation of carbon dioxide $\left(\mathrm{CO}_{2}\right)$ into valuable chemicals has attracted much attention as $\mathrm{CO}_{2}$ is an abundant, inexpensive, and renewable C1 feedstock [1-7]. Compared to the industrial large-scale transformation of $\mathrm{CO}_{2}$ into bulk chemicals, such as urea, salicylic acid, carbonate, and methanol, the preparation of fine chemicals using $\mathrm{CO}_{2}$ as a synthetic unit remains scarce [8]. Therefore, the development of more efficient reactions to catalytically convert $\mathrm{CO}_{2}$ into various valuable compounds is of high relevance $[9,10]$. Among these strategies, cyclization reactions represent the most commonly used and powerful approach as heterocycles offer tremendous diversity and are widely present in biologically important molecules [11,12]. Since $\mathrm{CO}_{2}$ usually serves as an electrophile, it can be incorporated into heterocyclic com- pounds via the reaction with nitrogen [13-15], oxygen [16-20], or carbon nucleophiles [21-26].

1,3,4-Oxadiazin-2-ones are important structural subunits found in many pharmacologically potent and biologically active compounds. Those compounds can be used as selective $\mathrm{Ca}^{2+}$ sensitizers, anticonvulsants, anxiolytic, antiulcer agents [27,28], and useful 1,2-diaza-1,3-butadiene precursors for the construction of other fine chemicals [29]. Typically, 1,3,4-oxadiazin-2-ones are prepared by the reaction of cyclic carbonate with hydrazine or by using carbazates through laborious multistep procedures [30,31]. Therefore, the development of more efficient and atom-economical approaches using $\mathrm{CO}_{2}$ as the carboxylative reagent is highly desirable. As a continuation of our interest in catalytic transformations of $\mathrm{CO}_{2}$ into fine chemicals [32-34], herein we report a silver-catalyzed carboxylative cyclization of alkynic hydrazones with $\mathrm{CO}_{2}$ to give

\footnotetext{
* Corresponding author. Tel: +86-411-84986257; Fax: +86-411-84986256; E-mail: zhangwz@dlut.edu.cn

This work was supported by the National Natural Science Foundation of China (21878038), the Natural Science Foundation of Liaoning Province (20170540156), and the Program for Changjiang Scholars and Innovative Research Team in University (IRT-17R14).

DOI: S1872-2067(19)63352-8 | http://www.sciencedirect.com/science/journal/18722067 | Chin. J. Catal., Vol. 40, No. 8, August 2019
} 
1,3,4-oxadiazin-2-ones in good yield under mild reaction conditions.

\section{Experimental}

\subsection{General information}

Unless otherwise stated, all manipulations were performed using standard Schlenk techniques under dry nitrogen or $\mathrm{CO}_{2}$ atmosphere. DMF and DMSO were distilled under $\mathrm{N}_{2}$ atmosphere with $\mathrm{CaH}_{2}$. $\mathrm{CH}_{3} \mathrm{CN}$ was distilled with $\mathrm{P}_{2} \mathrm{O}_{5}$. THF was distilled from sodium/benzophenone. All solvents were stored over $4 \AA ̊$ molecular sieves before use. Column chromatography was performed on silica gel (200-300 mesh). Thin layer chromatography was performed on $0.20 \mathrm{~mm}$ GF254 plates, which were visualized under UV light $(254 \mathrm{~nm})$. Unless otherwise stated, $\mathrm{CO}_{2}$ (99.999\%), commercial silver, copper, and palladium salts, and various bases were used without further purification.

NMR spectra were recorded on a $400 \mathrm{M}$ or $500 \mathrm{M}\left({ }^{1} \mathrm{H}\right.$ NMR, 400 or $500 \mathrm{MHz}$; ${ }^{13} \mathrm{C} \mathrm{NMR}, 101$ or $126 \mathrm{MHz}$ ) spectrometer in $\mathrm{CDCl}_{3}$ at ambient temperature, and the chemical shifts are expressed in parts per million $(\delta, \mathrm{ppm})$. The proton chemical shifts have been referenced to $7.26 \mathrm{ppm}\left(\mathrm{CHCl}_{3}\right)$ or $2.50 \mathrm{ppm}$ (DMSO) and carbon chemical shifts to $77.0 \mathrm{ppm}\left(\mathrm{CHCl}_{3}\right)$ or 39.5 ppm (DMSO). The data is reported using the following abbreviations: s, singlet; d, doublet; $\mathrm{t}$, triplet; $\mathrm{m}$, multiplet; hept, heptet; and $J$, coupling constant in Hz. High resolution mass spectra (HRMS) were recorded on a Q-TOF mass spectrometer equipped with a Z-spray ionization source. Infrared (IR) spectra were measured using a Nicolet NEXUS FT-IR spectrophotometer.

Substrates $\mathbf{1}$ and $\mathbf{4}$ were prepared according to reported procedures [35].

\subsection{General procedure for the carboxylative cyclization of alkynic hydrazones with $\mathrm{CO}_{2}$}

A $20 \mathrm{~mL}$ oven-dried autoclave containing a stir bar was charged with hydrazone (0.30 mmol), $\mathrm{Cs}_{2} \mathrm{CO}_{3}$ ( $4.9 \mathrm{mg}, 5 \mathrm{~mol} \%$ ), AgOAc (2.5 mg, $5 \mathrm{~mol} \%$ ), and $3.0 \mathrm{~mL}$ DMSO in a glove box. After removal from the glove box, the autoclave was purged with $\mathrm{CO}_{2}$ three times and then pressurized at $2.0 \mathrm{MPa} \mathrm{CO}_{2}$. The reaction mixture was stirred at $25^{\circ} \mathrm{C}$ for $2 \mathrm{~h}$, after which the remaining gas was vented slowly in the hood. The reaction mixture was diluted with $10 \mathrm{~mL}$ water and extracted with diethyl ether $(3 \times$ $10 \mathrm{~mL}$ ). The combined organic phase was washed with brine ( 2 $\times 10 \mathrm{~mL}$ ), dried over anhydrous $\mathrm{Na}_{2} \mathrm{SO}_{4}$, and concentrated under reduced pressure. The residue was purified by flash column chromatography on silica gel using petroleum/ethyl acetate as the eluent to afford the following oxadiazinone products.

(Z)-6-Benzylidene-3-(2-hydroxyethyl)-3,6-dihydro-2H-1,3,4 -oxadiazin-2-one (2a). White solid $(0.30 \mathrm{mmol}$ scale, $59 \mathrm{mg}$, $85 \%$ yield). $R_{\mathrm{f}}=0.4$ (EtOAc/petroleum ether $\left.=2: 1\right) .{ }^{1} \mathrm{H} \mathrm{NMR}$ (400 MHz, $\left.\mathrm{CDCl}_{3}\right): \delta 7.68(\mathrm{~d}, J=7.3 \mathrm{~Hz}, 2 \mathrm{H}), 7.36(\mathrm{t}, J=7.4 \mathrm{~Hz}$, $2 \mathrm{H}), 7.32-7.30(\mathrm{~m}, 1 \mathrm{H}), 7.23(\mathrm{~s}, 1 \mathrm{H}), 5.76(\mathrm{~s}, 1 \mathrm{H}), 3.97-3.92(\mathrm{~m}$, 4H), 2.59 (s, 1H) ppm. ${ }^{13} \mathrm{C}$ NMR (101 MHz, $\left.\mathrm{CDCl}_{3}\right): \delta 144.52$,
141.09, 138.07, 132.29, 129.68, 128.73, 128.64, 113.05, 60.39, 53.01 ppm. HRMS (ESI, $m / z$ ) calculated for $\mathrm{C}_{12} \mathrm{H}_{13} \mathrm{~N}_{2} \mathrm{O}_{3}[\mathrm{M}+\mathrm{H}]^{+}$: 233.0926, found: 233.0924. IR (neat): $v 3443,2925,1732,1275$ $\mathrm{cm}^{-1}$.

(Z)-6-Benzylidene-3-(2-cyanoethyl)-3,6-dihydro-2H-1,3,4oxadiazin-2-one (2b). White solid $(0.30 \mathrm{mmol}$ scale, $56 \mathrm{mg}$, $77 \%$ yield). $R_{\mathrm{f}}=0.3($ EtOAc/petroleum ether $=1: 1) .{ }^{1} \mathrm{H} \mathrm{NMR}$ (400 MHz, $\mathrm{CDCl}_{3}$ ): $\delta 7.68(\mathrm{~d}, J=7.6 \mathrm{~Hz}, 2 \mathrm{H}), 7.43-7.28(\mathrm{~m}, 4 \mathrm{H})$, $5.82(\mathrm{~s}, 1 \mathrm{H}), 4.06(\mathrm{t}, J=6.8 \mathrm{~Hz}, 2 \mathrm{H}), 2.79(\mathrm{t}, J=6.8 \mathrm{~Hz}, 2 \mathrm{H}) \mathrm{ppm}$. ${ }^{13} \mathrm{C}$ NMR $\left(101 \mathrm{MHz}, \mathrm{CDCl}_{3}\right): \delta 143.62,140.74,138.57,132.03$, 129.78, 128.98, 128.67, 116.89, 114.00, 46.59, 16.38 ppm. HRMS (ESI, $m / z$ ) calculated for $\mathrm{C}_{13} \mathrm{H}_{12} \mathrm{~N}_{3}[\mathrm{M}+\mathrm{H}]^{+}:$242.0930, found: 242.0929. IR (neat): $v 2924,2250,1738,1630 \mathrm{~cm}^{-1}$.

(Z)-6-Benzylidene-3-isopropyl-3,6-dihydro-2 $\mathrm{H}$-1,3,4-oxadiazin -2-one (2c). White solid ( $0.30 \mathrm{mmol}$ scale, $48 \mathrm{mg}, 69 \%$ yield. $R \mathrm{f}$ $=0.5($ EtOAc/petroleum ether $=1: 5) .{ }^{1} \mathrm{H} \mathrm{NMR}(400 \mathrm{MHz}$, $\left.\mathrm{CDCl}_{3}\right): \delta 7.70(\mathrm{~d}, J=7.6 \mathrm{~Hz}, 2 \mathrm{H}), 7.37(\mathrm{t}, J=7.5 \mathrm{~Hz}, 2 \mathrm{H})$, 7.32-7.24 (m, 2H), $5.73(\mathrm{~s}, 1 \mathrm{H}), 4.70$ (p, J = 6.7 Hz, 1H), 1.30 (d, $J$ $=6.7 \mathrm{~Hz}, 6 \mathrm{H})$ ppm. ${ }^{13} \mathrm{C}$ NMR $\left(101 \mathrm{MHz}, \mathrm{CDCl}_{3}\right): \delta 143.57,141.21$, 137.59, 132.67, 129.57, 128.64, 128.45, 111.85, 50.46, 19.96 ppm. HRMS (ESI, $m / z$ ) calculated for $\mathrm{C}_{13} \mathrm{H}_{14} \mathrm{~N}_{2} \mathrm{NaO}_{2}[\mathrm{M}+\mathrm{Na}]^{+}$: 253.0953, found: 253.0953. IR (neat): $v 3443,2921,1732 \mathrm{~cm}^{-1}$.

(Z)-3-Benzyl-6-benzylidene-3,6-dihydro-2H-1,3,4-oxadiazin -2-one (2d). White solid ( $0.30 \mathrm{mmol}$ scale, $75 \mathrm{mg}$, $90 \%$ yield). $R_{\mathrm{f}}$ $=0.4($ EtOAc/petroleum ether $=1: 5) .{ }^{1} \mathrm{H}$ NMR $(400 \mathrm{MHz}$, $\left.\mathrm{CDCl}_{3}\right): \delta 7.70(\mathrm{~d}, J=7.5 \mathrm{~Hz}, 2 \mathrm{H}), 7.45-7.31(\mathrm{~m}, 8 \mathrm{H}), 7.23(\mathrm{~s}, 1 \mathrm{H})$, $5.76(\mathrm{~s}, 1 \mathrm{H}), 4.94(\mathrm{~s}, 2 \mathrm{H}) \mathrm{ppm} .{ }^{13} \mathrm{C}$ NMR $\left(101 \mathrm{MHz}, \mathrm{CDCl}_{3}\right): \delta$ $144.03,141.39,138.09,135.97,132.43,129.70,128.70,128.64$, 128.55, 128.53, 128.08, 112.85, 54.55 ppm. HRMS (ESI, $m / z$ ) calculated for $\mathrm{C}_{17} \mathrm{H}_{15} \mathrm{~N}_{2} \mathrm{O}_{2}[\mathrm{M}+\mathrm{H}]^{+}:$279.1134, found: 279.1130 . IR (neat): $v 3424,2914,1732 \mathrm{~cm}^{-1}$.

(Z)-6-(4-Fluorobenzylidene)-3-(2-hydroxyethyl)-3,6-dihydro -2H-1,3,4-oxadiazin-2-one (2e). White solid (0.30 mmol scale, $52 \mathrm{mg}, 69 \%$ yield). $R_{\mathrm{f}}=0.3$ (EtOAc/petroleum ether $\left.=2: 1\right) \cdot{ }^{1} \mathrm{H}$ NMR (400 MHz, $\mathrm{CDCl}_{3}$ ): $\delta 7.67-7.63(\mathrm{~m}, 2 \mathrm{H}), 7.21(\mathrm{~s}, 1 \mathrm{H}), 7.03$ $(\mathrm{t}, J=8.7 \mathrm{~Hz}, 2 \mathrm{H}), 5.71(\mathrm{~s}, 1 \mathrm{H}), 3.95-3.90(\mathrm{~m}, 4 \mathrm{H}), 2.63(\mathrm{~s}, 1 \mathrm{H})$ ppm. ${ }^{13} \mathrm{C}$ NMR (101 MHz, $\left.\mathrm{CDCl}_{3}\right): \delta 162.54(\mathrm{~d}, J=251.49 \mathrm{~Hz})$, $144.43,140.77,137.93,131.54$ (d, $J=8.1 \mathrm{~Hz}), 128.57$ (d, $J=4.1$ Hz), 115.75 (d, $J=22.2 \mathrm{~Hz}$ ), 111.77, 60.29, 53.02 ppm. HRMS (ESI, $m / z$ ) calculated for $\mathrm{C}_{12} \mathrm{H}_{11} \mathrm{FN}_{2} \mathrm{NaO}_{3}[\mathrm{M}+\mathrm{Na}]^{+}:$273.0651, found: 273.0652. IR (neat): $v 3430,2921,1720 \mathrm{~cm}^{-1}$.

(Z)-6-(4-Chlorobenzylidene)-3-(2-hydroxyethyl)-3,6-dihydro -2H-1,3,4-oxadiazin-2-one (2f). White solid $(0.30 \mathrm{mmol}$ scale, $61 \mathrm{mg}, 76 \%$ yield). $R_{\mathrm{f}}=0.3$ (EtOAc/petroleum ether $\left.=2: 1\right) \cdot{ }^{1} \mathrm{H}$ NMR (400 MHz, $\left.\mathrm{CDCl}_{3}\right): \delta 7.61(\mathrm{~d}, J=8.4 \mathrm{~Hz}, 2 \mathrm{H}), 7.32(\mathrm{~d}, J=8.3$ $\mathrm{Hz}, 2 \mathrm{H}), 7.23$ (s, $J=10.0 \mathrm{~Hz}, 1 \mathrm{H}), 5.72(\mathrm{~s}, 1 \mathrm{H}), 3.96-3.94(\mathrm{~m}$, $4 \mathrm{H}), 2.37$ (s, $1 \mathrm{H}) \mathrm{ppm} .{ }^{13} \mathrm{C}$ NMR (126 MHz, $\left.\mathrm{CDCl}_{3}\right): \delta 144.31$, $141.47,137.79,134.60,130.88,130.85,128.96,111.76,60.61$, 53.16 ppm. HRMS (ESI, $m / z$ ) calculated for $\mathrm{C}_{12} \mathrm{H}_{12} \mathrm{ClN}_{2} \mathrm{O}_{3}$ $[\mathrm{M}+\mathrm{H}]^{+}:$267.0536, found: 267.0533. IR (neat): $v$ 3358, 2923, $1656 \mathrm{~cm}^{-1}$.

(Z)-3-(2-Hydroxyethyl)-6-(4-methylbenzylidene)-3,6-dihydro$2 \mathrm{H}-1,3,4-$ oxadiazin-2-one $(\mathbf{2 g})$. White solid $(0.30 \mathrm{mmol}$ scale, $52 \mathrm{mg}, 71 \%$ yield). $R \mathrm{f}=0.4$ (EtOAc/petroleum ether $=2: 1) \cdot{ }^{1} \mathrm{H}$ NMR (400 MHz, $\left.\mathrm{CDCl}_{3}\right): \delta 7.58(\mathrm{~d}, J=8.1 \mathrm{~Hz}, 2 \mathrm{H}), 7.21(\mathrm{~s}, 1 \mathrm{H})$, 7.17 (d, $J=8.0 \mathrm{~Hz}, 2 \mathrm{H}), 5.74(\mathrm{~s}, 1 \mathrm{H}), 3.96-3.93(\mathrm{~m}, 4 \mathrm{H}), 2.53$ (s, 1H), $2.35(\mathrm{~s}, 3 \mathrm{H}) \mathrm{ppm} .{ }^{13} \mathrm{C}$ NMR $\left(101 \mathrm{MHz}, \mathrm{CDCl}_{3}\right) \delta 144.71$, 
140.60, 139.06, 138.26, 129.69, 129.53, 129.41, 113.25, 60.51, 52.99, 21.37 ppm. HRMS (ESI, $m / z$ ) calculated for $\mathrm{C}_{13} \mathrm{H}_{15} \mathrm{~N}_{2} \mathrm{O}_{3}$ $[\mathrm{M}+\mathrm{H}]^{+}:$247.1083, found: 247.1077. IR (neat): $v$ 3430, 3054, $1728,1464 \mathrm{~cm}^{-1}$.

(Z)-3-(2-Hydroxyethyl)-6-(4-methoxybenzylidene)-3,6-dihy dro-2H-1,3,4-oxadiazin-2-one (2h). White solid $(0.30 \mathrm{mmol}$ scale, $63 \mathrm{mg}, 80 \%$ yield). $R_{\mathrm{f}}=0.4(\mathrm{EtOAc} /$ petroleum ether $=$ 2:1). ${ }^{1} \mathrm{H}$ NMR (400 MHz, $\mathrm{CDCl}_{3}$ ): $\delta 7.67(\mathrm{~d}, J=8.8 \mathrm{~Hz}, 2 \mathrm{H}$ ), 7.22 (s, 1H), 6.91 (d, $J=8.8 \mathrm{~Hz}, 2 \mathrm{H}), 5.76(\mathrm{~s}, 1 \mathrm{H}), 3.99-3.96(\mathrm{~m}, 4 \mathrm{H})$, $3.84(\mathrm{~s}, 3 \mathrm{H}) \mathrm{ppm} .{ }^{13} \mathrm{C}$ NMR $\left(101 \mathrm{MHz}, \mathrm{CDCl}_{3}\right): \delta 159.99,144.84$, 139.83, 138.42, 131.39, 125.14, 114.21, 113.11, 60.77, 55.29, 52.99 ppm. HRMS (ESI, $m / z$ ) calculated for $\mathrm{C}_{13} \mathrm{H}_{15} \mathrm{~N}_{2} \mathrm{O}_{4}[\mathrm{M}+\mathrm{H}]^{+}$: 263.1032, found: 263.1028. IR (neat): $v$ 3437, 3065, 1719, $1594,1275 \mathrm{~cm}^{-1}$.

(Z)-3-(2-Hydroxyethyl)-6-pentylidene-3,6-dihydro-2H-1,3,4 -oxadiazin-2-one (2i). Colorless liquid $(0.30 \mathrm{mmol} \mathrm{scale,} 50 \mathrm{mg}$, $78 \%$ yield). $R_{\mathrm{f}}=0.4$ (EtOAc/petroleum ether $\left.=2: 1\right) .{ }^{1} \mathrm{H} \mathrm{NMR}$ (400 MHz, $\left.\mathrm{CDCl}_{3}\right): \delta 7.08(\mathrm{~s}, 1 \mathrm{H}), 5.05-5.01(\mathrm{~m}, 1 \mathrm{H}), 3.93-3.88$ (m, 4H), 2.23-2.19 (m, 2H), 1.40-1.30 (m, 4H), 0.91-0.87 (m, $3 \mathrm{H}) \mathrm{ppm} .{ }^{13} \mathrm{C}$ NMR $\left(101 \mathrm{MHz}, \mathrm{CDCl}_{3}\right): \delta 145.57,141.77,137.51$, $116.17,60.26,52.68,30.56,23.75,22.16,13.66$ ppm. HRMS (ESI, $m / z$ ) calculated for $\mathrm{C}_{10} \mathrm{H}_{17} \mathrm{~N}_{2} \mathrm{O}_{3}[\mathrm{M}+\mathrm{H}]^{+}: 213.1239$, found: 213.1235. IR (neat): $v 3448,2956,1724,1654 \mathrm{~cm}^{-1}$.

(Z)-6-Hexylidene-3-(2-hydroxyethyl)-3,6-dihydro-2H-1,3,4oxadiazin-2-one (2j). Colorless liquid $(0.30 \mathrm{mmol} \mathrm{scale}, 53 \mathrm{mg}$, $78 \%$ yield). $R_{\mathrm{f}}=0.4$ (EtOAc/petroleum ether $\left.=2: 1\right) .{ }^{1} \mathrm{H} \mathrm{NMR}$ (400 MHz, $\left.\mathrm{CDCl}_{3}\right): \delta 7.06(\mathrm{~s}, 1 \mathrm{H}), 5.01(\mathrm{t}, J=7.8 \mathrm{~Hz}, 1 \mathrm{H}), 3.85(\mathrm{~s}$, $4 \mathrm{H}), 2.73(\mathrm{~s}, 1 \mathrm{H}), 2.20-2.14(\mathrm{~m}, 2 \mathrm{H}), 1.39-1.24(\mathrm{~m}, J=44.2 \mathrm{~Hz}$, $6 \mathrm{H}), 0.85(\mathrm{t}, J=6.9 \mathrm{~Hz}, 3 \mathrm{H}) \mathrm{ppm} .{ }^{13} \mathrm{C} \mathrm{NMR}\left(101 \mathrm{MHz}, \mathrm{CDCl}_{3}\right): \delta$ $145.58,141.76,137.53,116.26,60.33,52.68,31.26,28.13$, 24.02, 22.26, $13.82 \mathrm{ppm}$. HRMS (ESI, $\mathrm{m} / z$ ) calculated for $\mathrm{C}_{11} \mathrm{H}_{19} \mathrm{~N}_{2} \mathrm{O}_{3}[\mathrm{M}+\mathrm{H}]^{+}:$227.1396, found: 227.1386. IR (neat): $v$ $3440,2926,1724,1652 \mathrm{~cm}^{-1}$.

(Z)-6-(5-Chloropentylidene)-3-(2-hydroxyethyl)-3,6-dihydro -2H-1,3,4-oxadiazin-2-one (2k). White solid $(0.30 \mathrm{mmol}$ scale, $55 \mathrm{mg}, 75 \%$ yield). $R_{\mathrm{f}}=0.3(\mathrm{EtOAc} /$ petroleum ether $=2: 1) \cdot{ }^{1 \mathrm{H}}$ NMR (400 MHz, $\left.\mathrm{CDCl}_{3}\right): \delta 7.09(\mathrm{~s}, 1 \mathrm{H}), 5.01(\mathrm{t}, J=7.8 \mathrm{~Hz}, 1 \mathrm{H})$, $3.87(\mathrm{~s}, 4 \mathrm{H}), 3.52(\mathrm{t}, J=6.5 \mathrm{~Hz}, 2 \mathrm{H}), 2.58(\mathrm{~s}, 1 \mathrm{H}), 2.26-2.21(\mathrm{~m}$, 2H), $1.81-1.52(\mathrm{~m}, 4 \mathrm{H}) \mathrm{ppm} .{ }^{13} \mathrm{C}$ NMR (101 MHz, $\left.\mathrm{CDCl}_{3}\right): \delta$ 145.43, 142.18, 137.31, 115.00, 60.41, 52.76, 44.54, 31.88, 25.73, $23.32 \mathrm{ppm}$. HRMS (ESI, $m / z$ ) calculated for $\mathrm{C}_{10} \mathrm{H}_{16} \mathrm{ClN}_{2} \mathrm{O}_{3}$ $[\mathrm{M}+\mathrm{H}]^{+}:$247.0849, found: 247.0845. IR (neat): $v$ 3450, 2935, $1727,1654 \mathrm{~cm}^{-1}$.

(Z)-3-(2-Hydroxyethyl)-6-(2-phenoxyethylidene)-3,6-dihydro -2H-1,3,4-oxadiazin-2-one (2l). White solid (0.30 mmol scale, $45 \mathrm{mg}, 57 \%$ yield). $R_{\mathrm{f}}=0.3$ (EtOAc/petroleum ether $\left.=2: 1\right) .{ }^{1} \mathrm{H}$ NMR (400 MHz, $\left.\mathrm{CDCl}_{3}\right): \delta 7.32-7.27(\mathrm{~m}, 2 \mathrm{H}), 7.18(\mathrm{~s}, 1 \mathrm{H})$, 6.99-6.89 (m, 3H), $5.30(\mathrm{t}, J=6.4 \mathrm{~Hz}, 1 \mathrm{H}), 4.78(\mathrm{~d}, J=6.4 \mathrm{~Hz}$, $2 \mathrm{H}), 3.95-3.91(\mathrm{~m}, 4 \mathrm{H}), 2.02(\mathrm{~s}, 1 \mathrm{H}) \mathrm{ppm} .{ }^{13} \mathrm{C}$ NMR (126 MHz, $\left.\mathrm{CDCl}_{3}\right): \delta 157.92,144.58,142.70,136.26,129.56,121.28$, $114.56,109.90,60.79,60.40,53.08$ ppm. HRMS (ESI, $m / z$ ) calculated for $\mathrm{C}_{13} \mathrm{H}_{14} \mathrm{~N}_{2} \mathrm{NaO}_{4}[\mathrm{M}+\mathrm{Na}]^{+}:$285.0851, found: 285.0851 . IR (neat): $v 3408,2924,1737,1236 \mathrm{~cm}^{-1}$.

(Z)-6-Benzylidene-3,6-dihydro-2H-1,3,4-oxadiazin-2-one (5a). Pale white solid ( $0.30 \mathrm{mmol} \mathrm{scale}, 47 \mathrm{mg}, 83 \%$ yield). $R_{\mathrm{f}}=$ 0.3 (EtOAc/petroleum ether $=1: 2) .{ }^{1} \mathrm{H}$ NMR $\left(400 \mathrm{MHz}, \mathrm{CDCl}_{3}\right)$ : $\delta 8.17(\mathrm{~s}, 1 \mathrm{H}), 7.71(\mathrm{~d}, J=7.6 \mathrm{~Hz}, 2 \mathrm{H}), 7.41-7.31(\mathrm{~m}, 3 \mathrm{H}), 7.24(\mathrm{~s}$,
1H), 5.85 (s, 1H) ppm. ${ }^{13} \mathrm{C}$ NMR (126 MHz, $\left.\mathrm{CDCl}_{3}\right): \delta$ 154.38, $149.91,143.79,141.00,138.09,132.15,129.88,128.99,128.75$, 114.15 ppm. HRMS (ESI, $m / z$ ) calculated for $\mathrm{C}_{10} \mathrm{H}_{9} \mathrm{~N}_{2} \mathrm{O}_{2}[\mathrm{M}+\mathrm{H}]^{+}$: 189.0664, found: 189.0654. IR (neat): $v 2921,1727,1630 \mathrm{~cm}^{-1}$.

(Z)-6-(4-Fluorobenzylidene)-3,6-dihydro-2H-1,3,4-oxadiazin -2-one (5b). White solid ( $0.30 \mathrm{mmol}$ scale, $54 \mathrm{mg}, 87 \%$ yield). $R_{\mathrm{f}}$ $=0.3($ EtOAc/petroleum ether $=1: 2) .{ }^{1} \mathrm{H}$ NMR $(500 \mathrm{MHz}$, $\mathrm{CDCl}_{3}$ ): $\delta 8.15(\mathrm{~s}, 1 \mathrm{H}), 7.72-7.69(\mathrm{~m}, 2 \mathrm{H}), 7.22(\mathrm{~s}, 1 \mathrm{H}), 7.09-7.06$ $(\mathrm{m}, 2 \mathrm{H}), 5.81(\mathrm{~s}, 1 \mathrm{H}) \mathrm{ppm} .{ }^{13} \mathrm{C}$ NMR $\left(126 \mathrm{MHz}, \mathrm{CDCl}_{3}\right): \delta 162.77$ (d, $J=252 \mathrm{~Hz}), 143.65,140.67,137.95,131.77$ (d, $J=7.6 \mathrm{~Hz})$ ), 128.44, (d, $J=3.8 \mathrm{~Hz}$ ), 115.90 (d, $J=22.7 \mathrm{~Hz}$ ), 112.75 ppm. HRMS (ESI, $m / z$ ) calculated for $\mathrm{C}_{10} \mathrm{H}_{6} \mathrm{FN}_{2} \mathrm{O}_{2}[\mathrm{M}-\mathrm{H}]-:$ : 205.0413, found: 205.0420. IR (neat): $v 2925,1729,1590 \mathrm{~cm}^{-1}$.

(Z)-6-(4-Chlorobenzylidene)-3,6-dihydro-2H-1,3,4-oxadiazin -2-one (5c). White solid ( $0.30 \mathrm{mmol} \mathrm{scale,} 53 \mathrm{mg}, 79 \%$ yield). $R_{\mathrm{f}}$ $=0.3($ EtOAc/petroleum ether $=1: 2) .{ }^{1} \mathrm{H}$ NMR $(500 \mathrm{MHz}$, $\left.\mathrm{CDCl}_{3}\right): \delta 8.05(\mathrm{~s}, 1 \mathrm{H}), 7.64(\mathrm{~d}, J=8.5 \mathrm{~Hz}, 2 \mathrm{H}), 7.35(\mathrm{~d}, J=8.6 \mathrm{~Hz}$, 2H), 7.23 (s, 1H), 5.80 (s, 1H) ppm. ${ }^{13} \mathrm{C}$ NMR $\left(126 \mathrm{MHz}, \mathrm{CDCl}_{3}\right)$ : $\delta 143.40,141.28,137.79,134.83,131.02,130.67,129.02$, 112.63 ppm. HRMS (ESI, $m / z$ ) calculated for $\mathrm{C}_{10} \mathrm{H}_{6} \mathrm{ClN}_{2} \mathrm{O}_{2}$ [M-H]-: 221.0118, found: 221.0131. IR (neat): $v$ 2933, 1737, $1599 \mathrm{~cm}^{-1}$.

(Z)-6-(4-Methylbenzylidene)-3,6-dihydro-2H-1,3,4-oxadiazin -2-one (5d). White solid ( $0.30 \mathrm{mmol}$ scale, $52 \mathrm{mg}$, $85 \%$ yield). $R_{\mathrm{f}}$ $=0.3($ EtOAc/petroleum ether $=1: 2) .{ }^{1} \mathrm{H}$ NMR $(500 \mathrm{MHz}$, $\left.\mathrm{CDCl}_{3}\right): \delta 8.19(\mathrm{~s}, 1 \mathrm{H}), 7.61(\mathrm{~d}, J=8.1 \mathrm{~Hz}, 2 \mathrm{H}), 7.21-7.18(\mathrm{~m}, 3 \mathrm{H})$, $5.82(\mathrm{~s}, 1 \mathrm{H}), 2.37$ (s, 3H) ppm. ${ }^{13} \mathrm{C}$ NMR $\left(126 \mathrm{MHz}, \mathrm{CDCl}_{3}\right): \delta$ $144.03,140.47,139.32,138.25,129.86,129.50,129.40,114.13$, 21.44 ppm. HRMS (ESI, $m / z$ ) calculated for $\mathrm{C}_{11} \mathrm{H}_{9} \mathrm{~N}_{2} \mathrm{O}_{2}[\mathrm{M}-\mathrm{H}]^{-}$: 201.0664, found: 201.0679. IR (neat): $v 2924,1763,1616 \mathrm{~cm}^{-1}$.

(Z)-6-(4-Methoxybenzylidene)-3,6-dihydro-2H-1,3,4-oxadiazin -2-one (5e). Pale white solid $(0.30 \mathrm{mmol}$ scale, $41 \mathrm{mg}, 63 \%$ yield). $R_{\mathrm{f}}=0.2(\mathrm{EtOAc} /$ petroleum ether $=1: 2) .{ }^{1} \mathrm{H}$ NMR $(400$ $\left.\mathrm{MHz}^{\mathrm{CDCl}} \mathrm{CD}_{3}\right): \delta 8.02(\mathrm{~s}, 1 \mathrm{H}), 7.67(\mathrm{~d}, J=8.6 \mathrm{~Hz}, 2 \mathrm{H}), 7.19(\mathrm{~s}, 1 \mathrm{H})$, $6.91(\mathrm{~d}, J=8.6 \mathrm{~Hz}, 2 \mathrm{H}), 5.80(\mathrm{~s}, 1 \mathrm{H}), 3.84(\mathrm{~s}, 3 \mathrm{H}) \mathrm{ppm} .{ }^{13} \mathrm{C}$ NMR $\left(101 \mathrm{MHz}, \mathrm{CDCl}_{3}\right): \delta 160.12,144.07,139.68,138.35,131.53$, $124.97,114.24,113.86,55.32 \mathrm{ppm}$. HRMS (ESI, $m / z$ ) calculated for $\mathrm{C}_{11} \mathrm{H}_{9} \mathrm{~N}_{2} \mathrm{O}_{3}[\mathrm{M}-\mathrm{H}]^{-}:$: 217.0613, found: 217.0625. IR (neat): $v$ $2930,1755,1611,1147 \mathrm{~cm}^{-1}$.

(Z)-6-(Biphenylmethylene)-3,6-dihydro-2H-1,3,4-oxadiazin2-one (5f). White solid ( $0.30 \mathrm{mmol}$ scale, $49 \mathrm{mg}, 62 \%$ yield). $R_{\mathrm{f}}$ $=0.3($ EtOAc/petroleum ether $=1: 2) .{ }^{1} \mathrm{H}$ NMR $(400 \mathrm{MHz}$, $\left.\mathrm{CDCl}_{3}\right): \delta 8.05(\mathrm{~s}, 1 \mathrm{H}), 7.79(\mathrm{~d}, J=8.1 \mathrm{~Hz}, 2 \mathrm{H}), 7.64-7.61(\mathrm{~m}, 5 \mathrm{H})$, 7.48-7.35 (m, 3H), 5.89 (s, 1H) ppm. ${ }^{13} \mathrm{C}$ NMR (101 MHz, $\left.\mathrm{CDCl}_{3}\right): \delta 143.71,141.59,141.05,140.19,138.09,131.19$, $130.35,128.88,127.74,127.34,127.01,113.66$ ppm. HRMS (ESI, $m / z$ ) calculated for $\mathrm{C}_{16} \mathrm{H}_{11} \mathrm{~N}_{2} \mathrm{O}_{2}[\mathrm{M}-\mathrm{H}]^{-}:$: 263.0821, found: 263.0834. IR (neat): $v 2922,1738,1635 \mathrm{~cm}^{-1}$.

(Z)-6-(Cyclohexylmethylene)-3,6-dihydro-2H-1,3,4-oxadiazin -2-one (5g). White solid ( $0.30 \mathrm{mmol}$ scale, $40 \mathrm{mg}$, 69\% yield). $R_{\mathrm{f}}$ $=0.3($ EtOAc/petroleum ether $=1: 2) .{ }^{1} \mathrm{H}$ NMR $(400 \mathrm{MHz}$, $\left.\mathrm{CDCl}_{3}\right): \delta 8.10(\mathrm{~s}, 1 \mathrm{H}), 7.03(\mathrm{~s}, 1 \mathrm{H}), 4.94(\mathrm{~d}, J=9.6 \mathrm{~Hz}, 1 \mathrm{H})$, 2.61-2.59 (m, 1H), 1.72-1.61 (m, $14.0 \mathrm{~Hz}, 5 \mathrm{H}), 1.34-1.28$ (m, $2 \mathrm{H}), 1.20-1.09(\mathrm{~m}, 3 \mathrm{H}) \mathrm{ppm} .{ }^{13} \mathrm{C}$ NMR $\left(101 \mathrm{MHz}, \mathrm{CDCl}_{3}\right): \delta$ 145.10, 140.28, 137.75, 122.46, 33.39, 32.09, 25.77, 25.41 ppm. HRMS (ESI, $m / z$ ) calculated for $\mathrm{C}_{10} \mathrm{H}_{13} \mathrm{~N}_{2} \mathrm{O}_{2}[\mathrm{M}-\mathrm{H}]^{-}:$: 193.0977, found: 193.0987. IR (neat): $v 2930,1762,1242 \mathrm{~cm}^{-1}$. 
(Z)-6-Hexylidene-3,6-dihydro-2H-1,3,4-oxadiazin-2-one (5h). White solid ( $0.30 \mathrm{mmol}$ scale, $39 \mathrm{mg}, 71 \%$ yield). $R_{\mathrm{f}}=0.3$ (EtOAc/petroleum ether $=1: 4) .{ }^{1} \mathrm{H}$ NMR $\left(400 \mathrm{MHz}, \mathrm{CDCl}_{3}\right): \delta$ $8.63(\mathrm{~s}, 1 \mathrm{H}), 7.07(\mathrm{~s}, 1 \mathrm{H}), 5.08(\mathrm{t}, J=7.7 \mathrm{~Hz}, 1 \mathrm{H}), 2.21(\mathrm{q}, J=7.4$ $\mathrm{Hz}, 2 \mathrm{H}), 1.43-1.29(\mathrm{~m}, 6 \mathrm{H}), 0.88(\mathrm{t}, J=6.3 \mathrm{~Hz}, 3 \mathrm{H}) \mathrm{ppm} .{ }^{13} \mathrm{C}$ NMR (101 MHz, $\left.\mathrm{CDCl}_{3}\right): \delta$ 145.37, 141.65, 137.52, 117.10, 31.32, 28.18, 24.02, 22.34, 13.91 ppm. HRMS (ESI, $m / z$ ) calculated for $\mathrm{C}_{9} \mathrm{H}_{13} \mathrm{~N}_{2} \mathrm{O}_{2}$ [M-H]-: 181.0977, found: 181.0988. IR (neat): $v 2924,1747,1659 \mathrm{~cm}^{-1}$.

(Z)-6-(4-Chlorobutylidene)-3,6-dihydro- $2 \mathrm{H}$-1,3,4-oxadiazin2-one (5i). White solid ( $0.30 \mathrm{mmol}$ scale, $33 \mathrm{mg}, 54 \%$ yield). $R_{\mathrm{f}}$ $=0.3($ EtOAc/petroleum ether $=1: 4) \cdot{ }^{1} \mathrm{H}$ NMR $(400 \mathrm{MHz}$, $\left.\mathrm{CDCl}_{3}\right): \delta 8.39(\mathrm{~s}, 1 \mathrm{H}), 7.09(\mathrm{~s}, 1 \mathrm{H}), 5.09(\mathrm{t}, J=7.8 \mathrm{~Hz}, 1 \mathrm{H}), 3.55$ $(\mathrm{t}, J=6.6 \mathrm{~Hz}, 2 \mathrm{H}), 2.40(\mathrm{q}, J=7.6 \mathrm{~Hz}, 2 \mathrm{H}), 1.91(\mathrm{p}, J=6.9 \mathrm{~Hz}, 2 \mathrm{H})$ ppm. ${ }^{13} \mathrm{C}$ NMR $\left(101 \mathrm{MHz}, \mathrm{CDCl}_{3}\right): \delta 144.79,142.45,137.09$, 114.50, 44.04, 31.28, 21.60 ppm. HRMS (ESI, $m / z$ ) calculated for $\mathrm{C}_{7} \mathrm{H}_{8} \mathrm{ClN}_{2} \mathrm{O}_{2}$ [M-H] $]^{-}: 187.0274$, found: 187.0290. IR (neat): $v$ $2930,1747,1659,1059 \mathrm{~cm}^{-1}$.

\section{Results and discussion}

\subsection{Optimization of reaction conditions}

Alkynic hydrazones were firstly synthesized by the reaction of alkynals with hydrazines according to reported procedures [35]. In an initial investigation, alkynic aryl hydrazone was found to react with $\mathrm{CO}_{2}$ to give very low yields of the cyclization product under different reaction conditions due to the low nucleophilic reactivity of the nitrogen atom. As a consequence, phenylpropynal 2-hydroxyethylhydrazone (1a) was chosen as the model substrate for the reaction with $\mathrm{CO}_{2}$ to identify the optimal conditions (Table 1).

Considering that silver(I) salts can function as an effective $\pi$-Lewis acid catalyst for alkyne activation in carboxylation and other reactions [36-41], various silver-based catalytic systems were screened. The reaction of $\mathbf{1 a}$ with $2 \mathrm{MPa} \mathrm{CO}_{2}$ using 5

Table 1

Optimization of the reaction conditions for the carboxylative cyclization of alkynic hydrazones with $\mathrm{CO}_{2}$.

\begin{tabular}{|c|c|c|c|}
\hline Entry & Catalyst (mol\%) & Base (equiv.) & Yield (\%) \\
\hline 1 & AgOAc (5) & $\mathrm{Cs}_{2} \mathrm{CO}_{3}(1.5)$ & 87 \\
\hline 2 & CuI (5) & $\mathrm{Cs}_{2} \mathrm{CO}_{3}(1.5)$ & 75 \\
\hline $3^{a}$ & $\mathrm{Pd}(\mathrm{OAc})_{2}(5)$ & $\mathrm{Cs}_{2} \mathrm{CO}_{3}(1.5)$ & $<1$ \\
\hline 4 & $\mathrm{AgOAc}(5)$ & $\mathrm{K}_{2} \mathrm{CO}_{3}(1.5)$ & 85 \\
\hline 5 & AgOAc (5) & CsF (1.5) & 77 \\
\hline 6 & AgOAc (5) & DBU (1.5) & 30 \\
\hline $7^{b}$ & AgOAc (5) & $\mathrm{KO}^{t} \mathrm{Bu}(1.5)$ & $<1$ \\
\hline $8^{b}$ & $\mathrm{AgOAc}(5)$ & $\mathrm{NaH}(1.5)$ & $<1$ \\
\hline $9 \mathrm{c}$ & $\mathrm{AgOAc}(5)$ & $\mathrm{Cs}_{2} \mathrm{CO}_{3}(1.5)$ & 52 \\
\hline $10^{\mathrm{d}}$ & $\mathrm{AgOAc}(5)$ & $\mathrm{Cs}_{2} \mathrm{CO}_{3}(1.5)$ & 0 \\
\hline 11 & AgOAc (5) & - & 75 \\
\hline 12 & AgOAc (5) & $\mathrm{Cs}_{2} \mathrm{CO}_{3}(0.05)$ & 85 \\
\hline 13 & - & $\mathrm{Cs}_{2} \mathrm{CO}_{3}(0.05)$ & $<1$ \\
\hline
\end{tabular}

Reaction conditions: $\mathbf{1 a}(0.3 \mathrm{mmol}), 3 \mathrm{~mL}$ DMSO, $2.0 \mathrm{MPa} \mathrm{CO}_{2}, 25^{\circ} \mathrm{C}, 2 \mathrm{~h}$, isolated yield. a $\mathbf{1 a}$ was fully recovered. ${ }^{\mathrm{b}} \mathrm{Non}$-carboxylative cyclization product 3a was obtained. ${ }^{c}$ Using a $\mathrm{CO}_{2}$ balloon. ${ }^{\mathrm{d}} \mathrm{In}$ the absence of $\mathrm{CO}_{2}$. mol\% AgOAc as the catalyst and 1.5 equivalent $\mathrm{Cs}_{2} \mathrm{CO}_{3}$ as the base in DMSO at $25^{\circ} \mathrm{C}$ was found to readily afford the carboxylative cyclization product 3,6-dihydro- $2 \mathrm{H}$-1,3,4-oxadiazin -2-one (2a) in $87 \%$ isolated yield (Table 1, entry 1). It should be noted that the geometry of the $\mathrm{C}=\mathrm{N}$ bond in hydrazone substrates had no effect on the reactivity, probably due to the rapid conversion of the two isomers $Z$-1a and $E$-1a. The structural assignment of the isolated product was further confirmed by single-crystal X-ray analysis of 2a [42]. As shown in Fig. 1, the newly formed $\mathrm{C}=\mathrm{C}$ bond in the product exhibits $Z$ configuration, the typical structure obtained from Ag-catalyzed electrophilic cyclizations. Other silver(I) salts such as $\mathrm{AgI}, \mathrm{AgBF}_{4}, \mathrm{Ag}_{2} \mathrm{CO}_{3}$, and $\mathrm{AgNO}_{3}$ displayed inferior catalytic performance than $\mathrm{AgO}$ Ac (see SI). CuI showed lower catalytic activity than AgOAc (entry 2), while $\mathrm{Pd}(\mathrm{OAc})_{2}$ afforded no carboxylated product and 1a was fully recovered (entry 3). The replacement of $\mathrm{Cs}_{2} \mathrm{CO}_{3}$ with $\mathrm{K}_{2} \mathrm{CO}_{3}$ or $\mathrm{CsF}$ resulted in a slight decrease in the yield of 2a (entries 4 and 5). Although DBU is frequently employed as an efficient base in many carboxylation reactions using $\mathrm{CO}_{2}$, only $30 \%$ yield of $\mathbf{2 a}$ was obtained when applied as the base (entry 6). Switching the base to $\mathrm{KO}{ }^{t} \mathrm{Bu}$ and $\mathrm{NaH}$ led to the exclusive formation of the non-carboxylative cyclization product pyrazole 3a (entries 7 and 8 ) $[35,43]$. The $\mathrm{CO}_{2}$ pressure had an obvious effect on the reaction. A lower yield of $\mathbf{2 a}$ was obtained when the reaction was conducted at low $\mathrm{CO}_{2}$ pressure (entry 9). The reaction did not proceed in the absence of $\mathrm{CO}_{2}$, indicating that the carbonyl moiety in the resulting product originates from $\mathrm{CO}_{2}$ rather than from the carbonate base (entry 10). Interestingly, $\mathbf{2 a}$ in $75 \%$ yield was still obtained in the absence of a base (entry 11), implying that hydrazone 1a itself may serve as a base in this process. To maintain the high efficiency of the reaction, a catalytic amount of base was added. The presence of $5 \mathrm{~mol} \% \mathrm{Cs}_{2} \mathrm{CO}_{3}$ was enough to achieve $85 \%$ yield of 2a (entry 11), which is comparable to the result using stoichiometric bases (entries 1 and 4). No product was observed in the absence of the catalyst, confirming the catalytic role of the silver(I) salt (entry 12). Finally, further screening experiments revealed DMSO as the optimal solvent for this reaction (see $\mathrm{SI}$ ).

\subsection{Substrate scope}

Under the optimized reaction conditions, the scope of the silver-catalyzed carboxylative cyclization with regard to alkynic hydrazone substrates was investigated (Table 2). In addition to phenylpropynal 2-hydroxyethylhydrazone (1a), other $N$-alkyl substituted hydrazones such as 2-cyanoethylhydrazone (1b),

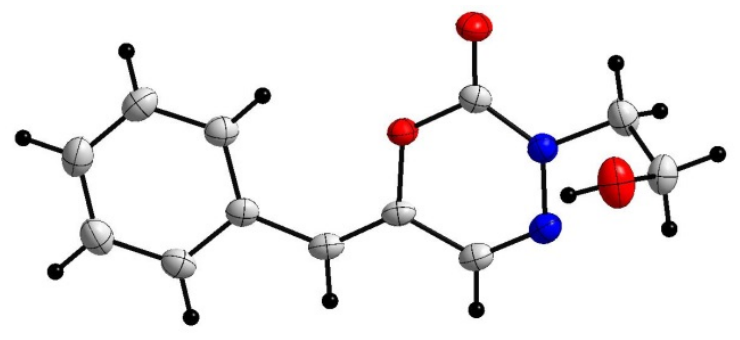

Fig. 1. Thermal ellipsoid plot for product $\mathbf{2 a}$. 
Table 2

Silver-catalyzed carboxylative cyclization of substituted alkynic hydrazones with $\mathrm{CO}_{2}$
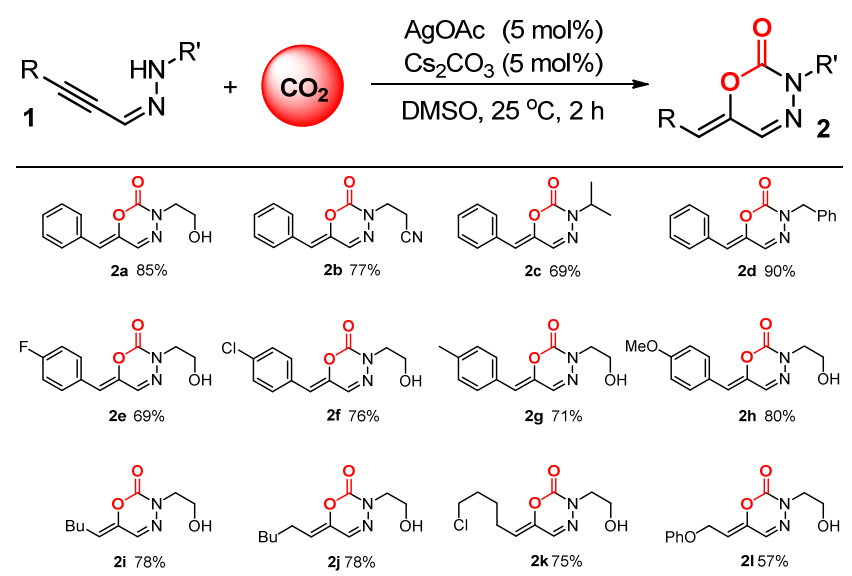

Reaction conditions: 1 (0.3 mmol), AgOAc (5 mol\%), $\mathrm{Cs}_{2} \mathrm{CO}_{3}(5 \mathrm{~mol} \%)$, $3 \mathrm{~mL}$ DMSO, $2.0 \mathrm{MPa} \mathrm{CO}_{2}$, reaction temperature $25^{\circ} \mathrm{C}$, reaction time $2 \mathrm{~h}$, isolated yield.

isopropylhydrazone (1c), and benzylhydrazone (1d) were found to be suitable substrates for this reaction. Arylpropynal 2-hydroxyethylhydrazones bearing various functional groups including electron-withdrawing (fluoro, chloro) and electron-donating (methyl, methoxyl) substituents reacted smoothly to afford the corresponding 3,6-dihydro-2H-1,3,4-oxadiazin-2-ones (2e-2h) in good yield. Alkyl-substituted propynal 2-hydroxyethylhydrazones 1i-1l participated efficiently in the carboxylative cyclization reaction furnishing the corresponding products in moderate to good yield.

Since nitrogen heterocycles in medicinal compounds frequently present $\mathrm{NH}$ moieties, many synthetic processes involve redundant nitrogen protection/deprotection steps. Therefore, carboxylative cyclization reactions of propynal hydrazone substrates $\mathbf{4}$ to give directly $\mathrm{N}$-unsubstituted products $\mathbf{5}$ were then examined (Table 3). Using the same reaction conditions to those applied to $N$-substituted hydrazone substrates $\mathbf{1}$, substrates $\mathbf{4}$ containing electron-withdrawing or electron-donating aryl and alkyl substituents on the alkyne moiety furnished the desired products $\mathbf{5}$ in satisfactory yield. The geometry of the newly formed $\mathrm{C}=\mathrm{C}$ bonds in oxadiazinones $\mathbf{5}$ was also identified as that of $Z$ isomers based on NOE experiments for products $\mathbf{5 a}$ and $\mathbf{5 g}$ (see SI).

This catalytic reaction is readily scalable for both types of substrates, as exemplified by the synthesis of 2 a ( $83 \%$ yield) and $\mathbf{5 a}$ (85\% yield) at gram scale with longer reaction times (Scheme 1). It should be mentioned that the isolated yield of $\mathbf{5 a}$ at $0.8 \mathrm{mmol}$ scale (Scheme 1) was slightly higher than the yield obtained at $0.3 \mathrm{mmol}$ scale (Table 3 ).

\subsection{Mechanistic studies}

In addition to the control experiments in Table 1 illustrating the catalytic role of silver(I) salts and the need for $\mathrm{CO}_{2}$ in this reaction, the reaction of $\mathbf{4 a}$ with $\mathrm{CO}_{2}$ in $\mathrm{DMSO}^{-} d_{6}$ was further

\section{Table 3}

Silver-catalyzed carboxylative cyclization of alkynic hydrazones with $\mathrm{CO}_{2}$.
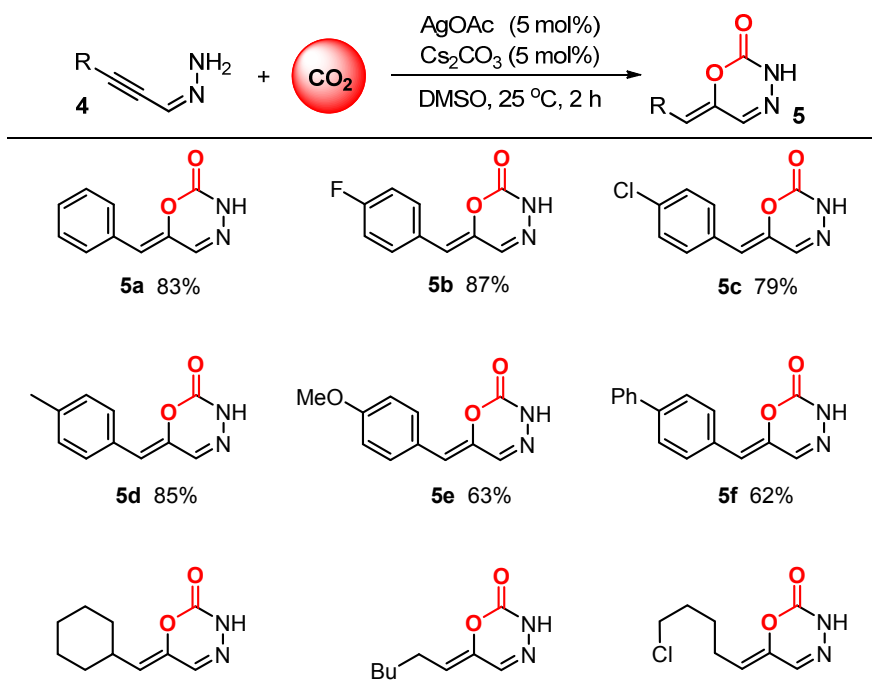

5g $69 \%$
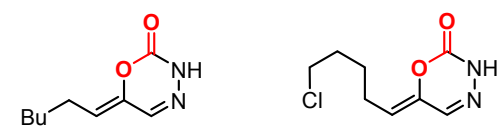

Reaction conditions: 4 (0.3 mmol), AgOAc (5 mol\%), $\mathrm{Cs}_{2} \mathrm{CO}_{3}(5 \mathrm{~mol} \%)$, $3 \mathrm{~mL}$ DMSO, $2.0 \mathrm{MPa} \mathrm{CO}_{2}$, reaction temperature $25^{\circ} \mathrm{C}$, reaction time $2 \mathrm{~h}$, isolated yield.

examined to detect possible reactive intermediates by NMR spectroscopy.

As shown in Fig. 2, the ${ }^{1} \mathrm{H}$ NMR spectrum of 4 a saturated with atmospheric $\mathrm{CO}_{2}$ in DMSO-d $d_{6}$ (Fig. 2(a)) was found to be identical to that of $\mathbf{4 a}$ without $\mathrm{CO}_{2}$, implying that the formation of a carbazate intermediate in the absence of an external base is inefficient despite the hydrazone itself being able to act as a base. When a catalytic amount of $\mathrm{Cs}_{2} \mathrm{CO}_{3}$ was added to the reaction system, two obvious new signals appeared at 6.26 and 8.23 ppm, assigned to the carbazate intermediate (Fig. 2(b)). The amount of this species gradually increased to a 1:4 ratio relative to 4a (Fig. 2(c)). When $10 \mathrm{~mol} \%$ of AgOAc was added to the reaction system, the signals for the carbazate intermediate and 4a began to disappear, while the signals of product 5a started to emerge (Fig. 2(d)). After $2 \mathrm{~h}$, the hydrazone substrate was completely converted into the oxadiazinone product and no obvious signals for the carbazate intermediate were detected anymore (Fig. 2(e)).

On the basis of the above experiments, a plausible mechanism for this reaction is proposed in Scheme 2. The Z-isomer of the hydrazone substrate enters a productive catalytic cycle where it interacts with the base. The reaction of the NH moiety of the hydrazone with $\mathrm{CO}_{2}$ affords the reactive carbazate intermediate $\mathbf{B}$. Then, the carboxylate oxygen atom conducts a nucleophilic attack on the silver(I)-activated alkyne moiety

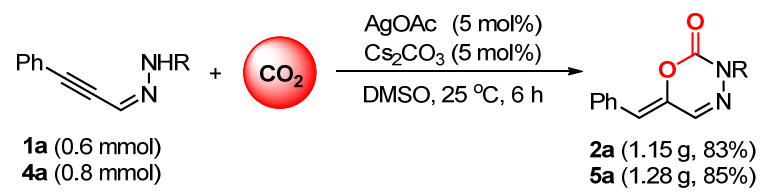

Scheme 1. Gram-scale reactions 


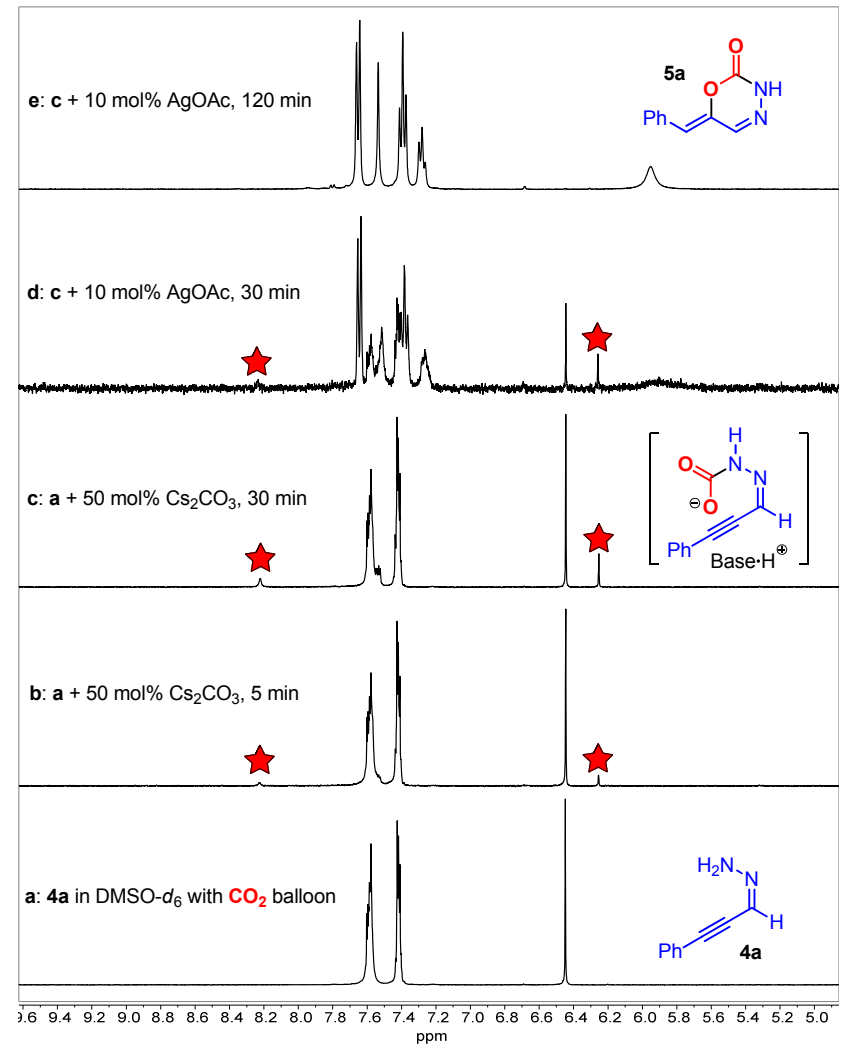

Fig. 2. NMR studies of the reaction of $\mathbf{4 a}$ with $\mathrm{CO}_{2}$.

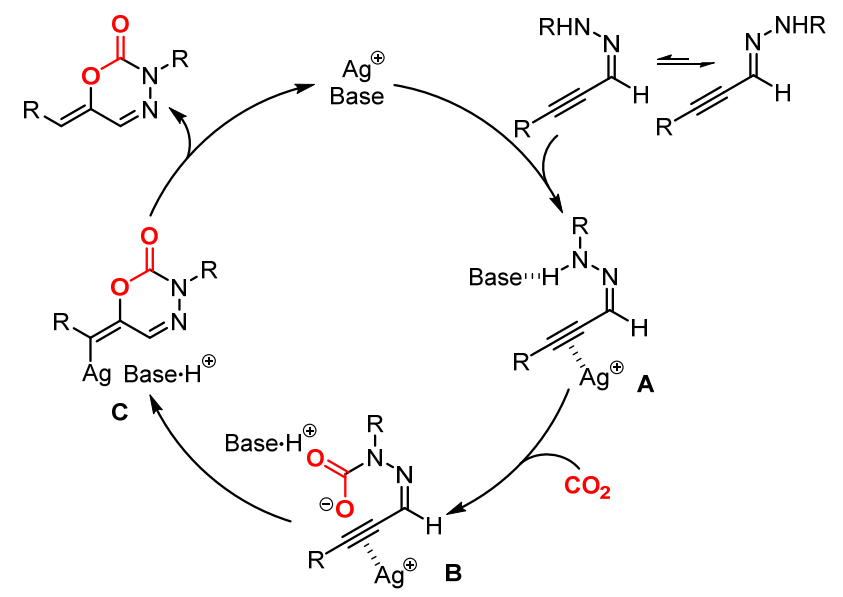

Scheme 2. Proposed mechanism.

affording vinylic silver intermediate $\mathbf{C}$. The following protonation reaction finally furnishes the carboxylative cyclization product, with concomitant regeneration of the catalyst and release of the base.

\section{Conclusions}

We have developed a silver(I)-catalyzed carboxylative cyclization of alkynic hydrazones under mild reaction conditions. Silver(I) salts show high activity in this reaction as $\pi$-Lewis acid catalysts for alkyne activation, and the use of catalytic amounts of a base such as cesium carbonate is sufficient to guarantee the rapid formation of the reactive carbazate intermediate. This reaction provides a highly efficient and convenient approach to synthesize biologically important 1,3,4-oxadiazin-2-one compounds in good yield using $\mathrm{CO}_{2}$ as the carboxylative reagent.

\section{References}

[1] X. B. Lu, D. J. Darensbourg, Chem. Soc. Rev., 2012, 41, 1462-1484.

[2] M. He, Y. Sun, B. Han, Angew. Chem. Int. Ed., 2013, 52, 9620-9633.

[3] C. Martin, G. Fiorani, A. W. Kleij, ACS Catal., 2015, 5, 1353-1370.

[4] L. Wang, W. Sun, C. Liu, Chin. J. Chem., 2018, 36, 353-362.

[5] J. Hao, W. Shi, Chin. J. Catal., 2018, 39, 1157-1166.

[6] S. S. Yan, Q. Fu, L. L. Liao, G. Q. Sun, J. H. Ye, L. Gong, Y. Z. Bo-Xue, D. G. Yu, Coord. Chem. Rev., 2018, 374, 439-463.

[7] C. C. Yan, L. Lin, G. X. Wang, X. H. Bao, Chin. J. Catal., 2019, 40, 23-37.

[8] A. Tortajada, F. Julia-Hernandez, M. Borjesson, T. Moragas, R. Martin, Angew. Chem. Int. Ed., 2018, 57, 15948-15982.

[9] F. Tan, G. Yin, Chin. J. Chem., 2018, 36, 545-554.

[10] Q. Liu, L. Wu, R. Jackstell, M. Beller, Nat. Commun., 2015, 6, 5933.

[11] B. Yu, L. N. He, ChemSusChem, 2015, 8, 52-62.

[12] S. Wang, C. Xi, Chem. Soc. Rev., 2019, 48, 382-404.

[13] J. H. Ye, L. Song, W. J. Zhou, T. Ju, Z. B. Yin, S. S. Yan, Z. Zhang, J. Li, D. G. Yu, Angew. Chem. Int. Ed., 2016, 55, 10022-10026.

[14] M. Y. Wang, Y. Cao, X. Liu, N. Wang, L. N. He, S. H. Li, Green Chem., 2017, 19, 1240-1244.

[15] S. Wang, X. Zhang, C. Cao, C. Chen, C. Xi, Green Chem., 2017, 19, 4515-4519.

[16] B. A. Vara, T. J. Struble, W. Wang, M. C. Dobish, J. N. Johnston, J. Am. Chem. Soc., 2015, 137, 7302-7305.

[17] J. Rintjema, R. Epping, G. Fiorani, E. Martin, E. C. Escudero-Adan, A. W. Kleij, Angew. Chem. Int. Ed., 2016, 55, 3972-3976.

[18] W. Y. Zhang, R. C. Luo, Q. H. Xu, Y. J. Chen, X. W. Lin, X. T. Zhou, H. B. Ji, Chin. J. Catal., 2017, 39, 736-744.

[19] X. Fu, X. Jing, L. Jin, L. Zhang, X. Zhang, B. Hu, H. Jing, Chin. J. Catal., 2018, 39, 997-1003.

[20] Q. W. Song, P. Liu, L. H. Han, K. Zhang, L. N. He, Chin. J. Chem., 2018, $36,147-152$.

[21] J. Hou, A. Ee, W. Feng, J. Xu, Y. Zhao, J. Wu, J. Am. Chem. Soc., 2018, $140,5257-5263$.

[22] Z. Zhang, C. J. Zhu, M. Miao, J. L. Han, T. Ju, L. Song, J. H. Ye, J. Li, D. G. Yu, Chin. J. Chem., 2018, 36, 430-436.

[23] L. Fu, S. Li, Z. Cai, Y. Ding, X. Guo, L. Zhou, D. Yuan, Q. Sun, G. Li, Nat. Catal., 2018, 1, 469-478.

[24] X. Gao, B. Yu, Z. Yang, Y. Zhao, H. Zhang, L. Hao, B. Han, Z. Liu, ACS Catal., 2015, 5, 6648-6652.

[25] Z. Zhang, Q. Sun, C. Xia, W. Sun, Org. Lett., 2016, 18, 6316-6319.

[26] D. Zhu, L. Fang, H. Han, Y. Wang, J. Xia, Org. Lett., 2017, 19, 4259-4262.

[27] M. C. Forest, P. Lahouratate, M. Martin, G. Nadler, M. J. Quiniou, R. G. Zimmermann, J. Med. Chem., 1992, 35, 163-172.

[28] Y. Gohee, A. Boucherle, M. Robin, Eur. J. Med. Chem., 1986, 21, 403-409.

[29] R. K. Boeckman Jr, P. Ge, J. E. Reed, Org. Lett., 2001, 3, 3647-3650.

[30] R. M. Wilson, T. J. Chow, Tetrahedron Lett., 1983, 24, 4635-4636.

[31] D. Geffken, F. K. Hansen, M. Khankischper, T. Mauz, R. Meurer, Synlett, 2012, 23, 637-639.

[32] C. X. Guo, W. Z. Zhang, N. Zhang, X. B. Lu, J. Org. Chem., 2017, 82, 7637-7642.

[33] W. Z. Zhang, N. Zhang, Y. Q. Sun, Y. W. Ding, X. B. Lu, ACS Catal., 2017, 7, 8072-8076. 


\section{Graphical Abstract}

Chin. J. Catal., 2019, 40: 1153-1159 doi: S1872-2067(19)63352-8

\section{Silver-catalyzed carboxylative cyclization of alkynic hydrazones with carbon dioxide}

Wenzhen Zhang *, Yuqian Sun, Min Zhang, Hui Zhou, Xiaobing Lu Dalian University of Technology

A silver(I)-catalyzed carboxylative cyclization of alkynic hydrazones with carbon dioxide under mild reaction conditions was developed, which efficiently affords biologically important 1,3,4-oxadiazin-2-one compounds in good yield.

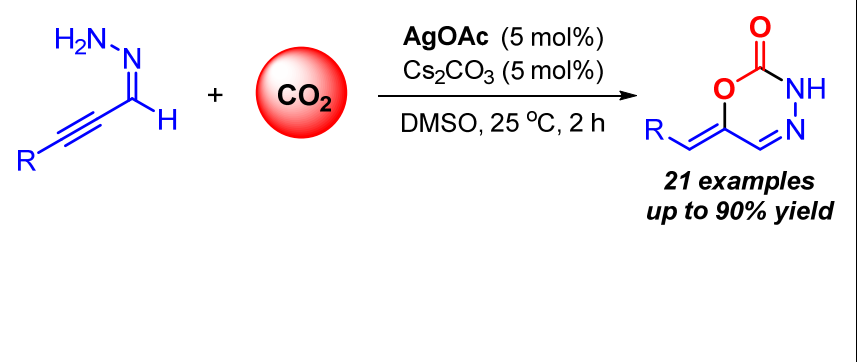

[40] C. Qi, D. Yan, W. Xiong, H. Jiang, J. CO Util., 2018, 24, 120-127.

[41] W. Wang, Y. Fu, Y. Li, R. Yao, L. Liu, W. Chang, J. Li, Org. Chem. Front., 2018, 5, 3331-3335.

[42] CCDC 1893129 contain the supplementary crystallographic data for product 2 a. These data can be obtained free of charge via www.ccdc.cam.ac.uk/data_request/cif.

[43] T. Kusakabe, H. Sagae, K. Kato, Org. Biomol. Chem., 2013, 11, 4943-4948.

\section{银催化炔基腙与二氧化碳的羧化环化反应}

张文珍", 孙玉乾, 张敏, 周 辉, 吕小兵 大连理工大学精细化工国家重点实验室, 辽宁大连 116024

摘要: 将二氧化碳作为羰一合成子催化转化成重要的杂环精细化学品的方法学研究受到广泛关注. 1,3,4-啞二嗪-2-酮类化 合物是一类具有重要生理活性的氮氧杂环化合物, 广泛用于医药如钙增敏剂、强心剂、杀菌及除草剂等. 传统的合成此类 化合物的方法包括环状碳酸酯与肼的反应、肼基甲酸酯化合物的闭环反应等, 其反应步骤长, 效率低, 不够经济绿色. 本文 开发了一种银催化的炔腙类化合物与二氧化碳羧化环化新反应用于高效合成1,3,4-噁二嗪-2-酮.

首先选取苯乙炔基羟乙基腙与二氧化碳的羧化环化反应作为模式反应, 笁选出适用于此反应的最优反应条件为: 5 $\mathrm{mol} \%$ 醋酸银为催化剂, $5 \mathrm{~mol} \%$ 碳酸铯为碱, 二氧化碳压力为 $2.0 \mathrm{MPa}$, 二甲基亚砜为溶剂, $25{ }^{\circ} \mathrm{C}$ 下应 $2 \mathrm{~h}$. 炔腙分子中碳氮 双键的顺反构型对其羧化反应活性没有影响. 当使用化学当量的叔丁醇钾或氢化钠等作为碱时, 即使在 $2.0 \mathrm{MPa}$ 的二氧化 碳存在下, 炔腙底物也几乎全部转化成非羧化闭环产物吡唑类化合物. 在最优反应条件下,氮原子上烷基取代或未取代的 炔腙底物能与二氧化碳高效发生羧化环化反应, 以 $54 \%-90 \%$ 的产率得到 20 多种 $1,3,4$-噁二嗪-2-酮产物. 反应放大到克级时 依旧能高效进行. 产物的X射线单晶衍射及氢核磁NOE实验证实, 新形成的碳碳双键为顺式结构. 当使用氮原子上芳基取 代的炔腙时, 由于底物中氮原子的亲核反应活性较差, 目标羧化产物产率很低.

一价银盐通常在二氧化碳参与的羧化反应中作为路易斯酸催化剂活化碳碳参键. 本文对照实验也证明在此羧化环化 反应中银盐具有不可或缺的催化作用. 改变反应气氛的实验也验证了1,3,4-噁二嗪-2-酮产物中的羰基来自二氧化碳. 氢核 磁跟踪实验观察到加入催化量碱能有效促进关键肼基甲酸根中间体的形成. 以此为依据, 本文提出了包含二氧化碳插入 炔腙底物形成肼基甲酸根、羧基氧亲核进攻银盐活化的碳碳参键形成烯基银中间体、质子化得到1,3,4-噁二嗪-2-酮产物及 再生银催化剂及碱的催化反应机理.

总之, 本文发展了一种银催化的简单易得的炔腙类化合物与二氧化碳的羧化环化新反应, 为 $1,3,4-$ 噁二嗪-2-酮类化合 物的合成提供了一种方便高效的方法.

关键词: 二氧化碳; 银催化; 羧化环化反应; 炔基腙; 啞二嗪酮; 均相催化

收稿日期: 2019-01-29. 接受日期: 2019-03-09. 出版日期: 2019-08-05.

*通讯联系人. 电话: (0411)84986257; 传真: (0411)84986256; 电子信箱: zhangwz@dlut.edu.cn 基金来源：国家自然科学基金(21878038); 辽宁省自然科学基金(20170540156); 长江学者和创新团队发展计划(IRT-17R14).

本文的电子版全文由Elsevier出版社在ScienceDirect上出版(http://www.sciencedirect.com/science/journal/18722067). 